\title{
An Application of Energy-Filtered Elemental Mapping Tomography in Semiconductor Device Analysis
}

\author{
Qiang Jin \\ Micron Technology, Inc., 8000 S. Federal Way, P.O. Box 6, Boise, Idaho 83707
}

As semiconductor devices continue to shrink, two-dimensional (2D) TEM imaging is often inadequate for analyzing complex non-planar features and production related defects. A full picture of the device structure requires advanced characterization with high spatial resolution in all three dimensions. As a technique of reconstructing a 3D structure from a series of 2D images, electron tomography has been extended in recent years from biology to many materials science applications. To avoid diffraction contrast in bright-field TEM imaging, both high-angle annular dark-field (HAADF) STEM [1] and energy-filtered TEM (EFTEM) [2] have been used in 3D volume reconstruction for highly crystalline materials. Despite the presence of weak diffraction contrast, energy-filtered elemental maps have been demonstrated to be suitable for $3 \mathrm{D}$ reconstruction by using $\mathrm{Cr}$ carbides at grain boundaries of a stainless steel [2]. In this paper, an example is given to demonstrate the application of energy-filtered elemental mapping tomography in semiconductor device characterization.

A tilt series was acquired from an aluminum metal line with $\mathrm{TiAl}_{3}$ particles at the interface with BPSG (boron phosphorus silicate glass) in a device specimen (Fig.1a). The data were collected in single-axis tilt sampling geometry. The $512 \times 512$ pixel EFTEM images were acquired using acquisition time of $12 \mathrm{~s}$ at a magnification of $46,000 \times$, resulting in a pixel size of $1.28 \mathrm{~nm}$ at the specimen level. The tilt series was obtained over a tilt range of $\pm 50^{\circ}$ at $2^{\circ}$ steps. At each tilt, three energy loss images, each with a $100 \mathrm{eV}$ window width, were acquired over the $\mathrm{Ti}_{2,3}$ edge (onset at $455.5 \mathrm{eV}$ ), two pre-edges at $400 \mathrm{eV}$ and $300 \mathrm{eV}$, and a post-edge at $510 \mathrm{eV}$. The three energy-loss images acquired at each tilt were aligned independently for shift relative to the first pre-edge image using a cross-correlation routine provided in Gatan DigitalMicrograph. An elemental map was generated from the three aligned images at each tilt. Inspect3D was used to align the tilt series of elemental maps by an iterative cross-correlation approach. The 3D volume reconstruction was carried out using SIRT (simultaneous iterative reconstruction technique) implemented in Inspect3D with 30 iterations. The reprojection of the $3 \mathrm{D}$ volume in the $0^{\circ}$ tilt direction is shown in Fig. $1 \mathrm{~b}$. Some of the $\mathrm{TiAl}_{3}$ structure obscured by diffraction contrast in the zero loss image is clearly shown in the Ti map projection. The voxel projections from the elemental map reconstruction are shown in Fig.2. These projections show clearly that $\mathrm{TiAl}_{3}$ particles are complex and irregular in shape along the length of the A1/BPSG interface. It is interesting to note that there exist some areas with brighter contrast among the $\mathrm{TiAl}_{3}$ particles. These might be unreacted Ti. Obviously, EFTEM tomography enables fine-scale 3D chemical distribution analysis. Otherwise, the complex 3D shapes and orientations of the $\mathrm{TiAl}_{3}$ particles can only be guessed from a single elemental map. This information should allow a better insight into the formation of $\mathrm{TiAl}_{3}$ and provide guidance for process control.

References

[1] C. Kübel, A. Voigt, R. Schoenmakers, M. Otten, D. Su, T.-C. Lee, A. Carlsson, and J. Bradley, Microsc. Microanal. 11(2005) 378.

[2] M.W. Weyland, P.A. Midgley, Microsc. Microanal. 9 (2003) 542. 

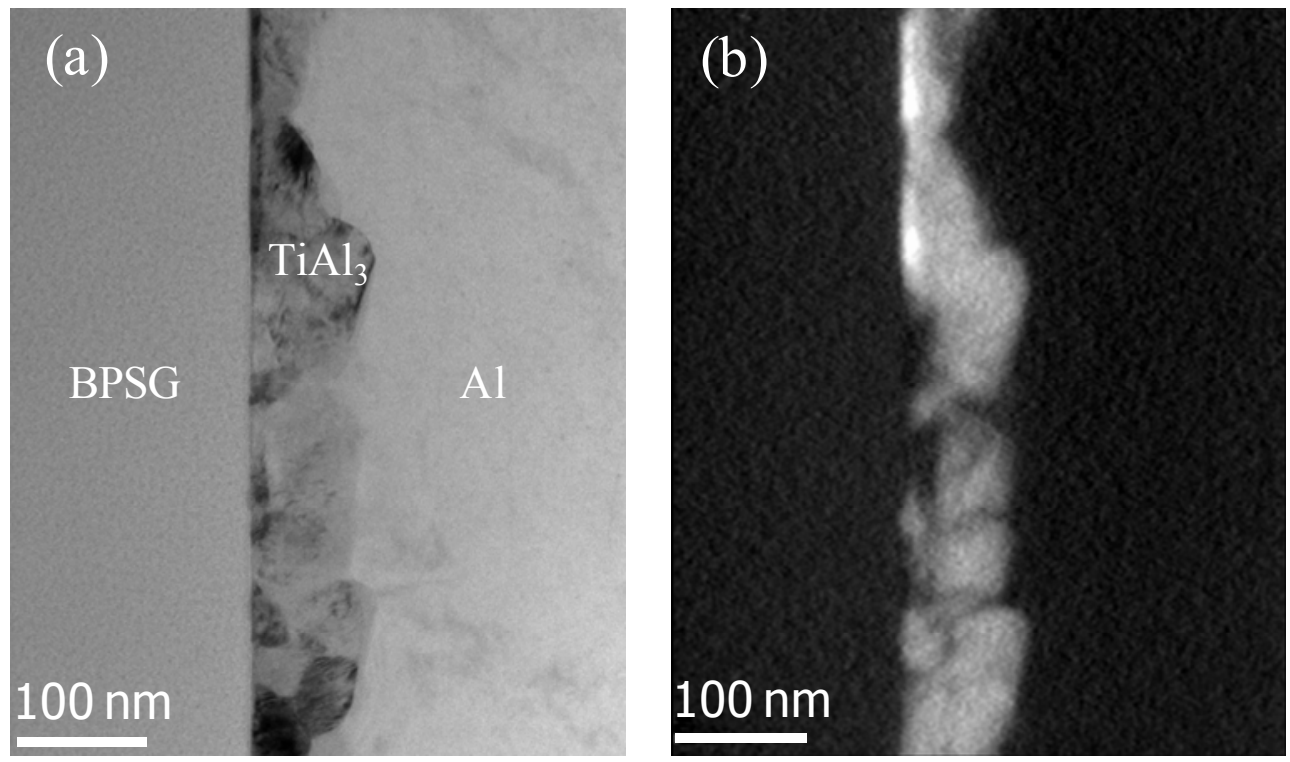

FIG.1. (a) Zero-loss image of the aluminum line with $\mathrm{TiAl}_{3}$ particles chosen for EFTE tomography, and (b) The reconstruction of Ti maps reprojected in the zero degree direction.

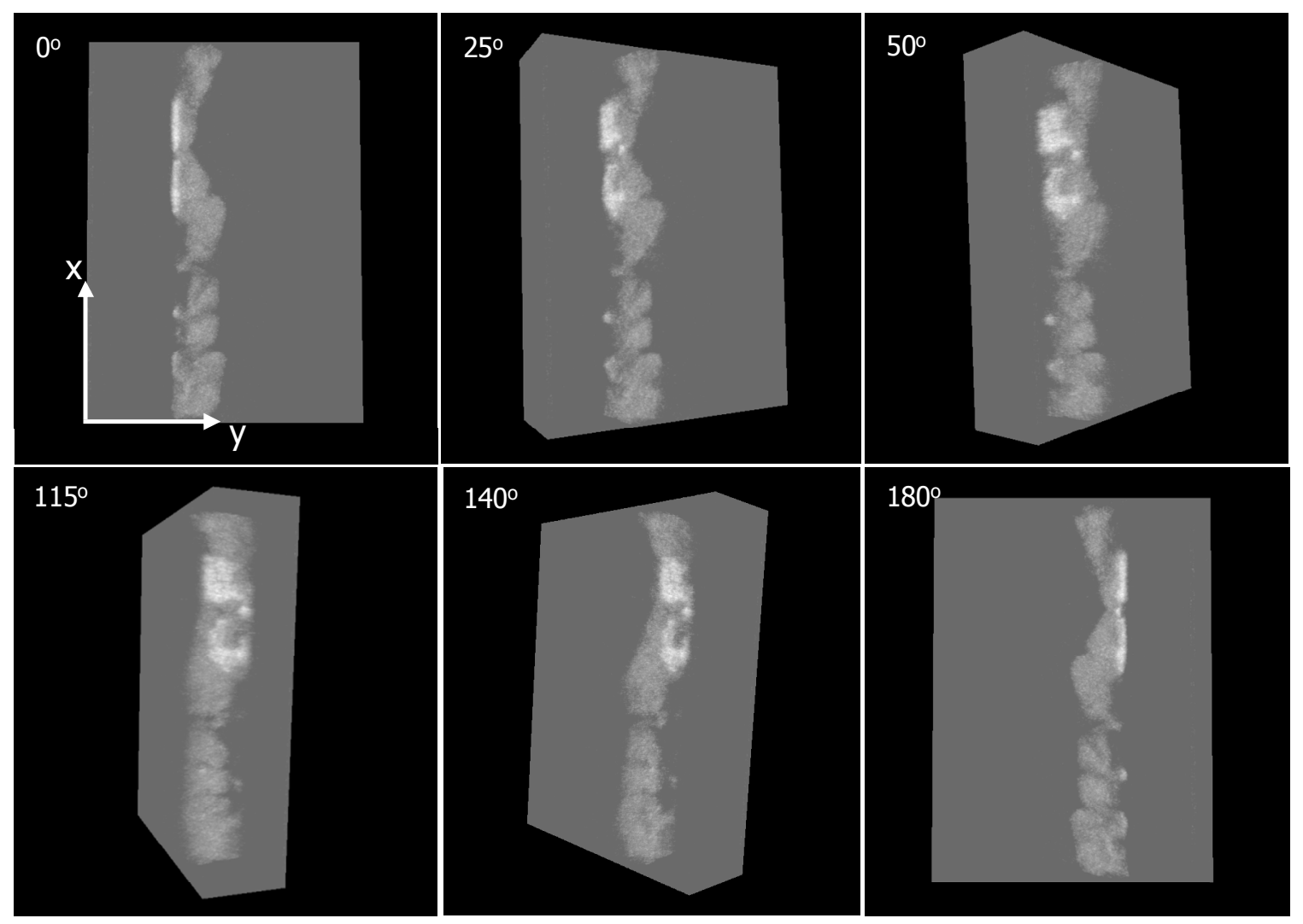

FIG. 2. Voxel projections from the tomographic reconstruction of the Ti maps. These have been contrast adjusted to show the distinctive shape of the $\mathrm{TiAl}_{3}$ particles. The number on the image indicates the angle rotated clockwise around the $\mathrm{x}$-axis. 